\title{
A Passivity-Based Controller for Motion Tracking and Damping Assignment for Compliantly Actuated Robots
}

\author{
Manuel Keppler ${ }^{1}$, Dominic Lakatos ${ }^{1}$, Christian Ott $^{1}$, Alin Albu-Schäffer ${ }^{1,2}$
}

\begin{abstract}
This paper introduces a novel control approach for motion tracking and damping assignment in compliantly actuated robotic systems. The approach follows the idea of shaping the link side dynamics such that the closed loop dynamics follows a given reference trajectory while injecting additional damping for improving vibration suppression. The modification of the apparent link side dynamics is amended by a desired dynamics on the motor side. In contrast to classical feedback linearization the design aims at only a minimal modification of the dynamics, both for the link side and the motor side. The time-varying closed loop dynamics is shown to be globally, uniformly stable. The effectiveness of the resulting feedback control law was evaluated by simulations as well as experiments on a highly nonlinear and highly compliant robot arm.
\end{abstract}

\section{INTRODUCTION}

Introducing compliant actuators in robotic systems improves mechanical robustness against rigid impacts as well as unknown contact forces and potentially increases the energetic efficiency [1]. The elastic elements acting between motor and link inertias lead to a dynamical behavior of a low-pass filter on external loads. In contrast to classical flexible-joint robots (FJR) where the linear elasticities are mainly a result of weight reduction, the generally statedependent stiffness of compliantly actuated robots is in an order of magnitude lower such that singular perturbation assumptions which allow to neglect the motor dynamics definitely do not hold any more. Mechanical compliance provides many benefits, but it also comes at a price; the plant dynamics are under-actuated as the number of dimensions of the configuration space is twice the number of dimensions of the control input space. Moreover, to improve energy storing capabilities and efficiency in general, compliant actuators are often designed such that damping and friction in parallel to the spring is negligible. Thus, unwanted intrinsic oscillatory dynamics may arise. In addition, many variable stiffness robots feature highly nonlinear elasticity, see e.g Fig. 1b. This is what makes control of the link configuration variables a challenging task.

Regulation controllers for the link configuration variables of flexible joint robots have been proposed in [2], [3], [4], [5] and a generalization to the case of nonlinear joint elasticities (often appearing in variable stiffness actuators (VSA) [6]) has been proposed in [7]. The above controllers consider only feedback of control-input-collocated variables. Therefore, the

\footnotetext{
${ }^{1}$ The authors are with the Institute of Robotics and Mechatronics, German Aerospace Center (DLR), D-82234 Oberpfaffenhofen, Germany. Contact: manuel.keppler@dlr.de

${ }^{2}$ Alin Albu-Schäffer is also with Technical University Munich, Chair of Sensor Based Robots and Intelligent Assistance Systems, Department of Informatics, D-85748 Garching, Germany.
}

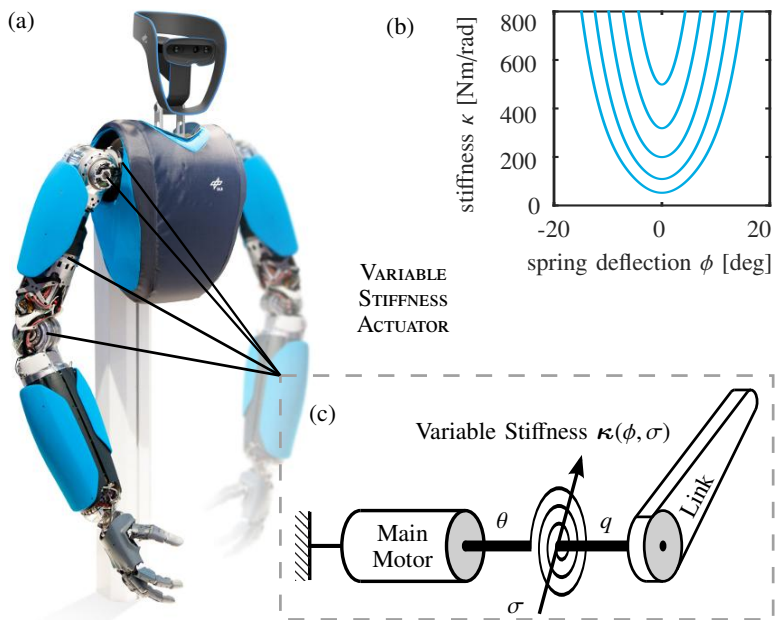

Fig. 1. Fig. 1a shows the DLR Hand Arm System. Fig. 1b shows a simplified working scheme of a single VSA. Fig. 1c depicts the stiffness characteristics for stiffness adjuster positions $\sigma=[0,2.5,5.0,7.5,10] \mathrm{deg}$, the outer curve corresponds to $\sigma=0 \mathrm{deg}$.

damping performance of these approaches is lower compared to the regulation controllers reported in [8], [9], [10] which feedback also control input non-collocated variables. While [8] provides a comprehensive stability analysis for constant controller gains, the closed-loop dynamics of [9] and [10] are not accompanied by a rigorous stability analysis.

Tracking controllers for the link configuration variables of robots with elastic transmissions are reported in the pioneering works [11], [12], [13]. Further solutions to the tracking problem are based on cascaded structures [14], integrator backstepping [15], [16, Chap. 6.2], extensions of the well known controller by Slotine and $\mathrm{Li}$ [17] to the flexible joint case [18], feedback linearization [11], [19], [20], and integral manifold control [11]. All of the tracking controllers above, [11]-[19], are formulated for linear elasticities with one exception: the method of [20] also applies to robots with nonlinear elasticities. Most of the tracking controllers above have been verified only in computer simulations.

We aim at deriving an effective tracking controller for the link configuration variables of compliantly actuated robots, with nonlinear elastic transmissions, that is theoretically well founded (stability proof) and practically relevant (validation by multi-joint experiments). In our previous work [?], we presented a solution to this problem by adding only damping and feedforward terms to the dynamics of the link variables with neither changing the rigid body dynamics of the links nor the structure of the nonlinear springs. By introducing new coordinates reflecting these damping and feedforward terms 
and a corresponding coordinate transformation of the motor dynamics, we achieved dynamics which structurally equals the dynamics of the original coordinates by feedback control. The desired tracking behavior (and disturbance rejection) of the link coordinates resulting from the original link dynamics with the additional desired damping and tracking terms have been implemented by pure PD regulation control in the new motor coordinates.

This paper further extends the concept of "minimalistic" feedback control to solve the tracking problem for the link configuration variables of compliantly actuated robots. This is done by avoiding the scaling of the motor inertia [21] in the new coordinates to constant values which is a nonpassive control action for itself. As a result of the further reduced control action, the experiments revealed that the control input (i.e., the motor torques) are reduced compared to our previous controller [?]. On the basis of the controller formulation introduced in this paper, it can be shown that in the limiting case of rigid actuation (i.e., stiffness approaches infinity) the classical PD+ controller [22] results. In contrast to many state of the art FJR controllers, no high-gain design results in this limiting case (cf. [23, chapt. 4.1]). The above properties, together with the stability analysis and the multijoint experiments, close the loop from theoretical foundations to experimental validation which to our best knowledge has not been done for tracking the configuration variables of compliantly actuated robots yet.

The paper is structured as follows: the idea is presented in Sec. II and the problem is formulated in Sec. III. Sec. IV proposes the controller design and Sect. V proves stability and passivity of the closed-loop dynamics. Finally, experimental validation is provided in Sec. VI and Sec. VII briefly concludes the work.

\section{A. Notation and Terminology}

Let $\overline{\boldsymbol{A}}$ and $\boldsymbol{A}$ denote the supremum and infimum of the Eigenvalues of a matrix $\boldsymbol{A}$. Throughout the text when talking about boundedness of matrices, we refer to it in the sense of bounded singular values. $\|$.$\| denotes the 2-norm (Euclidean$ norm) for vectors, and the induced 2-norm (spectral norm) for matrices.

\section{Design IDEA}

Consider a single robot joint with nonlinear flexible transmission as depicted in Fig. 2a. The control input is a generalized force $u$ acting on the motor inertia $B$ which drives the link inertia $M$ via an intermediate spring. In general, the generalized spring force $\psi(\phi)$ is a nonlinear function of the spring deflection $\phi=\theta-q$, where $\theta$ is the motor position and $q$ the link position.

We aim to find a control concept that exclusively adds link-side damping and tracking terms while changing the original dynamics to a minimal extent. By minimizing the dynamical shaping we except smaller feedback gains and therefore higher robustness with respect to noise and model uncertainties. To achieve this, we introduce new motor coordinates $\boldsymbol{\eta}$ that reflect the desired damping and tracking (a)
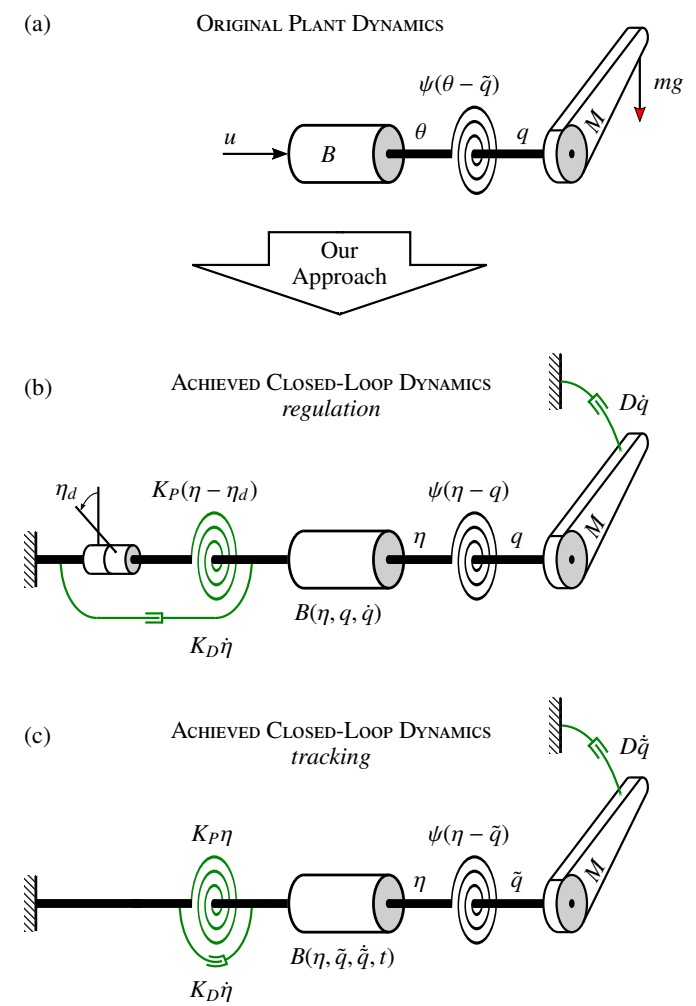

$\theta$ motor coord. $q$ link coord. $\tilde{q}$ tracking error $\tilde{q}=q-q_{d}(t)$ $q_{d}$ des. link pos.

Fig. 2. Design idea shown for a single joint with flexible transmission. The feedback control $\boldsymbol{u}$ adds link-side damping, regulation and tracking behavior, respectively, while changing the original dynamics only to a minimal extent. The concept relies on introducing new motor coordinates that reflect the desired link-side behavior. A graphical representation of the resulting closed-loop dynamics is shown.

behavior. For the regulation case we yield a closed-loop dynamic as depicted in 2 b. ${ }^{1}$ Note, the new link side dynamics behaves like the original one with compensated gravity but with additional damping and it is still connected via the same elastic transmission characterized by $\psi$. As we do a full coordinate transformation, a state-dependent, virtual motor inertia will arise. ${ }^{2}$ Since we aim to minimize dynamic shaping, we keep these terms intact instead of performing a feedback cancellation as in [24]. The newly introduced spring and damper to the left of the motor inertia stabilizes the system and drives it to the set point. For the regulation case, $\eta_{d}$ is a function of $q_{d}$. For the tracking case which is emphasized here, the resulting motor inertia due to the coordinate transformation will not only be state-dependent but also time-variant, see Fig. 2c. Note, the dynamics are written in error variables such that the problem is reduced to stabilization of the origin $\eta, \tilde{q} \rightarrow 0$, as visualized above.

\footnotetext{
${ }^{1}$ For ease of understanding we describe the concept for the regulation case first. The regulation case is not treated in the remainder of the paper, but the interested reader can simplify the proposed tracking controller to the straightforward case of regulation control.

${ }^{2}$ In Sec. IV we will see that also virtual, coordinate-dependent Coriolis terms appear.
} 


\section{Problem Statement}

Throughout this paper, we consider a simplified model of a $n$-link robot with compliant joints which is based on the model proposed by [11]. It assumes that the angular part of the kinetic energy of each rotor is due only to its own rotation, and is given by

$$
\begin{aligned}
M(\boldsymbol{q}) \ddot{\boldsymbol{q}}+\boldsymbol{C}(\boldsymbol{q}, \dot{\boldsymbol{q}}) \dot{\boldsymbol{q}} & =-\boldsymbol{g}(\boldsymbol{q})+\boldsymbol{\psi}(\boldsymbol{\theta}-\boldsymbol{q})+\tau_{\text {ext }} \\
\boldsymbol{B} \ddot{\boldsymbol{\theta}}+\boldsymbol{\psi}(\boldsymbol{\theta}-\boldsymbol{q}) & =\boldsymbol{u} .
\end{aligned}
$$

Herein, $\boldsymbol{q} \in \mathbb{R}^{n}$ and $\boldsymbol{\theta} \in \mathbb{R}^{n}$ represent the link angles and motor angles, respectively. $M \in \mathbb{R}^{n \times n}$ is the inertia matrix of the rigid links, $\boldsymbol{B} \in \mathbb{R}^{n \times n}$ is the diagonal matrix of actuator inertias. They have the following properties:

Property 1. The mass matrix $\boldsymbol{M ( q )}$ is symmetric, positive definite.

Property 2. The singular values of $\boldsymbol{M}(\boldsymbol{q})$ and $\boldsymbol{B}$ are bounded above and bounded below away from zero, thus both $M^{-1}$ and $\boldsymbol{B}^{-1}$ exist and are bounded.

These conditions are fulfilled for all pure rotational and pure prismatic joint robots and in some special cases for robots that feature a mix of rotational and prismatic joints, see [25] for an in-depth discussion. We denote the vector of Coriolis and centrifugal forces by $\boldsymbol{C}(\boldsymbol{q}, \dot{\boldsymbol{q}}) \dot{\boldsymbol{q}}, \boldsymbol{g}(\boldsymbol{q})$ represents the gravitational forces. As suggested by [18], we define $\boldsymbol{C}(\boldsymbol{q}, \dot{\boldsymbol{q}})$ via the Christoffel symbols, such that the model (1)(2) features the following properties:

Property 3. Since $\boldsymbol{C}(\boldsymbol{q}, \dot{\boldsymbol{q}})$ is bounded in $\boldsymbol{q}$ and linear in $\dot{\boldsymbol{q}}$, $\boldsymbol{C}$ is bounded for bounded $\dot{\boldsymbol{q}}$. The matrix $\dot{\boldsymbol{M}}(\boldsymbol{q})-2 \boldsymbol{C}(\boldsymbol{q}, \dot{\boldsymbol{q}})$ is skew symmetric for all $(\boldsymbol{q}, \dot{\boldsymbol{q}}) \in \mathbb{R}^{n} \times \mathbb{R}^{n}$.

Dynamics (1)-(2) represents an under-actuated mechanical system in which only the generalized motor coordinates $\boldsymbol{\theta}$ can be directly actuated via the generalized motor forces $\boldsymbol{u} \in$ $\mathbb{R}^{n}$. We consider $\boldsymbol{u}$ as the control input. The link coordinates $\boldsymbol{q}$ can only be indirectly actuated via the generalized elastic forces $\psi$ which are derived from the strictly convex and positive definite spring potential function $U_{s}(\phi)$. This form of under-actuation is the major challenge in the control of the states $\boldsymbol{q}, \dot{\boldsymbol{q}}$.

Assumption 1. The elastic potential function $U_{s}(\phi) \in C^{3}$ is strongly convex and positive definite for all $\phi \in \mathbb{R}^{n}$, i.e. $U_{s}(\phi)=0 \Longrightarrow \phi=0$. $^{3}$

Assumption 2. The generalized elastic torques $\psi(\phi)$ can be derived from the spring potential function $U_{s}$

$$
\boldsymbol{\psi}(\boldsymbol{\theta}-\boldsymbol{q}):=\left.\left(\frac{\partial U_{s}(\boldsymbol{\phi})^{T}}{\partial \boldsymbol{\phi}}\right)^{T}\right|_{\boldsymbol{\phi}=\boldsymbol{\theta}-\boldsymbol{q}} \in \mathbb{R}^{n}
$$

Strong convexity of $U_{s}$ ensures a constant $c>0$ exists such that

$$
\inf _{\phi \in \mathbb{R}^{n}} \lambda_{\min }\left(\frac{\partial \psi(\phi)}{\partial \phi}\right)>c, \quad \forall \phi \in \mathbb{R}^{n}
$$

\footnotetext{
${ }^{3}$ The strong convexity is no limiting condition from a practical point of view, as a lower bound for the joint stiffness is necessary such that the robot does not collapse under its gravitational weight and thus becomes uncontrollable.
}

Loosely speaking, $\boldsymbol{\psi}$ is strictly monotonic in its argument. As the Jacobian determinant of $\psi$ is nonzero due to (4), the inverse function theorem guarantees that $\psi$ is a global diffeomorphism. The local stiffness, the Hessian of the spring potential $U_{s}$, is denoted as follows

$$
\kappa\left(\phi_{0}\right):=\left.\left(\frac{\partial U_{s}(\phi)^{T}}{\partial \phi}\right)^{T}\right|_{\phi=\phi_{0}}=\left.\frac{\partial \psi(\phi)}{\partial \phi}\right|_{\phi=\phi_{0}} \in \mathbb{R}^{n \times n} .
$$

The generalized external foces which are exhibited by the manipulators environment are represented by $\tau_{\text {ext }}$.

In this paper we address the problem of finding a control input $\boldsymbol{u}$ which allows to enforce a desired link-side damping behavior, while simultaneously achieving link-side tracking.

\section{Controller Design}

\section{A. Desired Link-Side Dynamics}

Our goal is to achieve link-side tracking and damping behavior while changing the dynamics of the original system to a minimal extent. Therefore, we extend the link-side dynamics solely by a damping term and feed forward terms to ensure tracking performance. With that in mind, in the following we derive a control input $\boldsymbol{u}$ for (2), such that the link-side behavior of the resulting compensated system equals the following desired dynamics ${ }^{4}$

$$
\boldsymbol{M}(\tilde{\boldsymbol{q}}, t) \ddot{\tilde{\boldsymbol{q}}}+\boldsymbol{C}(\tilde{\boldsymbol{q}}, \dot{\tilde{\boldsymbol{q}}}, t) \dot{\tilde{\boldsymbol{q}}}=-D \dot{\tilde{\boldsymbol{q}}}+\boldsymbol{\psi}(\boldsymbol{\eta}-\tilde{\boldsymbol{q}})+\boldsymbol{\tau}_{\text {ext }},
$$

where $\tilde{\boldsymbol{q}}:=\boldsymbol{q}-\boldsymbol{q}_{d}(t) \in \mathbb{R}^{n}$ is the link-side tracking error. The desired trajectory has the following properties:

Assumption 3. $\boldsymbol{q}_{d}(t) \in C^{4}$, with $\left\|\boldsymbol{q}_{d}\right\|,\left\|\dot{\boldsymbol{q}}_{d}\right\|, \ldots,\left\|\boldsymbol{q}_{d}^{(4)}\right\|$ being bounded.

$\boldsymbol{\eta}$ are suitable motor coordinates, which will be introduced later, that reflect the desired link-dynamics. A desired linkside damping behavior, such that $\dot{\tilde{\boldsymbol{q}}} \rightarrow \mathbf{0}$, can be realized by an adequate choice of the damping matrix $D \in \mathbb{R}^{n \times n}$. It has to meet the following conditions:

Assumption 4. The damping matrix $\boldsymbol{D}$ is positive definite and bounded below such that $\underline{D}>0$, and it is bounded above such that $\bar{D}$ is finite.

We do not specify $\boldsymbol{D}$ any further here, as it is up to the user to design $\boldsymbol{D}$ as long as the assumptions above are fulfilled.

\section{B. Coordinate Transformation}

Note, the link-side of the new system (6) behaves like the original system (1) without gravity but with additional damping and tracking properties. We introduce new motor coordinates that reflect this behavior. To this end, we equate (1) and (6) to find an implicit relation

$$
\boldsymbol{\psi}(\boldsymbol{\theta}-\boldsymbol{q})-\boldsymbol{g}(\boldsymbol{q})=\boldsymbol{\psi}(\boldsymbol{\eta}-\tilde{\boldsymbol{q}})-\boldsymbol{D} \dot{\tilde{q}}-\boldsymbol{n}(\boldsymbol{q}, \dot{\boldsymbol{q}}, t)
$$

\footnotetext{
${ }^{4}$ With better readability in mind and to emphasize the explicit time dependency of the closed-loop link dynamics, we use the short forms $\boldsymbol{M}(\tilde{\boldsymbol{q}}, t)=\boldsymbol{M}\left(\tilde{\boldsymbol{q}}+\boldsymbol{q}_{d}(t)\right)=\boldsymbol{M}(\boldsymbol{q}), \boldsymbol{C}(\tilde{\boldsymbol{q}}, \dot{\tilde{\boldsymbol{q}}}, t)=\boldsymbol{C}\left(\tilde{\boldsymbol{q}}+\boldsymbol{q}_{d}(t), \dot{\tilde{\boldsymbol{q}}}+\dot{\boldsymbol{q}}_{d}(t)\right)=$ $\boldsymbol{C}(\boldsymbol{q}, \dot{\boldsymbol{q}})$ and $\boldsymbol{g}(\tilde{\boldsymbol{q}}, t)=\boldsymbol{g}\left(\tilde{\boldsymbol{q}}+\boldsymbol{q}_{d}(t)\right)=\boldsymbol{g}(\boldsymbol{q})$, respectively.
} 
between the new motor coordinates $\boldsymbol{\eta}$ and the original system states of (1)-(2), where

$$
\boldsymbol{n}(\tilde{\boldsymbol{q}}, \dot{\tilde{\boldsymbol{q}}}, t):=\boldsymbol{M}(\tilde{\boldsymbol{q}}, t) \ddot{\boldsymbol{q}}_{d}(t)+\boldsymbol{C}(\tilde{\boldsymbol{q}}, \dot{\tilde{\boldsymbol{q}}}, t) \dot{\boldsymbol{q}}_{d}(t) .
$$

Since the inverse of $\boldsymbol{\psi}$ is usually not analytically available, (7) cannot be solved directly and $\boldsymbol{\eta}$ has to be determined numerically. ${ }^{5}$ Differentiating (7) with respect to time gives us a differential relation between the old and new motor coordinates 6

$$
\boldsymbol{\kappa}(\boldsymbol{\theta}-\boldsymbol{q})(\dot{\boldsymbol{\theta}}-\dot{\boldsymbol{q}})-\dot{\boldsymbol{g}}(\boldsymbol{q})=\boldsymbol{\kappa}(\boldsymbol{\eta}-\tilde{\boldsymbol{q}})(\dot{\boldsymbol{\eta}}-\dot{\tilde{\boldsymbol{q}}})-\mathrm{D}_{t}(\boldsymbol{D} \dot{\tilde{\boldsymbol{q}}})+\dot{\boldsymbol{n}} .
$$

Solving (9) for $\dot{\boldsymbol{\theta}}$ gives us ${ }^{7}$

$$
\begin{aligned}
\dot{\boldsymbol{\theta}}= & \boldsymbol{\kappa}^{-1}(\boldsymbol{\theta}-\boldsymbol{q}) \boldsymbol{\kappa}(\boldsymbol{\eta}-\tilde{\boldsymbol{q}}) \dot{\boldsymbol{\eta}}+\dot{\tilde{\boldsymbol{q}}}+\dot{\boldsymbol{q}}_{d}(t) \\
& +\boldsymbol{\kappa}^{-1}(\boldsymbol{\theta}-\boldsymbol{q}) \gamma(\boldsymbol{\eta}, \tilde{\boldsymbol{q}}, \dot{\tilde{\boldsymbol{q}}}, \ddot{\tilde{\boldsymbol{q}}}, t),
\end{aligned}
$$

where

$$
\gamma(\boldsymbol{\eta}, \tilde{\boldsymbol{q}}, \dot{\tilde{\boldsymbol{q}}}, \ddot{\tilde{\boldsymbol{q}}}, t):=\dot{\boldsymbol{g}}(\tilde{\boldsymbol{q}}, t)-\boldsymbol{\kappa}(\boldsymbol{\eta}-\tilde{\boldsymbol{q}}) \dot{\tilde{\boldsymbol{q}}}-\mathrm{D}_{t}(\boldsymbol{D} \dot{\tilde{\boldsymbol{q}}})+\dot{\boldsymbol{n}}(\tilde{\boldsymbol{q}}, \dot{\tilde{\boldsymbol{q}}}, t)
$$

With (7) we can re-write $\dot{\theta}$ as a function of the new states only

$$
\dot{\boldsymbol{\theta}}=\boldsymbol{A}(\boldsymbol{\eta}, \tilde{\boldsymbol{q}}, \dot{\tilde{\boldsymbol{q}}}, t) \dot{\boldsymbol{\eta}}+\boldsymbol{a}(\boldsymbol{\eta}, \tilde{\boldsymbol{q}}, \dot{\tilde{\boldsymbol{q}}}, \ddot{\tilde{\boldsymbol{q}}}, t)
$$

See Appendix for $\boldsymbol{A}$ and $\boldsymbol{a}$. Differentiating (12) with respect to time yields

$$
\ddot{\boldsymbol{\theta}}=\boldsymbol{A}(\boldsymbol{\eta}, \tilde{\boldsymbol{q}}, \dot{\tilde{\boldsymbol{q}}}, t) \ddot{\boldsymbol{\eta}}+\dot{\boldsymbol{A}}(\boldsymbol{\eta}, \tilde{\boldsymbol{q}}, \dot{\tilde{\boldsymbol{q}}}, t) \dot{\boldsymbol{\eta}}+\dot{\boldsymbol{a}}(\boldsymbol{\eta}, \tilde{\boldsymbol{q}}, \dot{\tilde{\boldsymbol{q}}}, \ddot{\tilde{\boldsymbol{q}}}, t)
$$

The relations above allow us to make a full coordinate transformation $[\boldsymbol{\theta}, \boldsymbol{q}] \mapsto[\boldsymbol{\eta}, \tilde{\boldsymbol{q}}]$ for the robot dynamics (1)(2).

\section{New Motor Dynamics}

Substituting $\boldsymbol{\psi}(\boldsymbol{\theta}-\boldsymbol{q})$ and $\ddot{\boldsymbol{\theta}}$ from (7) and (13) into (2) and pre-multiplying by $\boldsymbol{A}^{\mathrm{T}}$ yields the transformed motor dynamics

$$
\begin{aligned}
& \boldsymbol{M}_{\eta}(\boldsymbol{\eta}, \tilde{\boldsymbol{q}}, \dot{\tilde{\boldsymbol{q}}}, t) \ddot{\boldsymbol{\eta}}+\boldsymbol{C}_{\eta}(\boldsymbol{\eta}, \dot{\boldsymbol{\eta}}, \tilde{\boldsymbol{q}}, \dot{\tilde{\boldsymbol{q}}}, \ddot{\tilde{\boldsymbol{q}}}, t) \dot{\boldsymbol{\eta}}+\boldsymbol{A}^{\mathrm{T}} \boldsymbol{\psi}(\boldsymbol{\theta}-\boldsymbol{q}) \\
& +\boldsymbol{A}^{\mathrm{T}}(\boldsymbol{\eta}, \tilde{\boldsymbol{q}}, \dot{\tilde{\boldsymbol{q}}}, t) \boldsymbol{B} \dot{\boldsymbol{a}}(\boldsymbol{\eta}, \dot{\boldsymbol{\eta}}, \tilde{\boldsymbol{q}}, \dot{\tilde{\boldsymbol{q}}}, \ddot{\tilde{\boldsymbol{q}}}, t)=\boldsymbol{A}^{\mathrm{T}} \boldsymbol{u},
\end{aligned}
$$

where

$$
\boldsymbol{M}_{\eta}(\boldsymbol{\eta}, \tilde{\boldsymbol{q}}, \dot{\tilde{\boldsymbol{q}}}, t):=\boldsymbol{A}^{\mathrm{T}}(\boldsymbol{\eta}, \tilde{\boldsymbol{q}}, \dot{\tilde{\boldsymbol{q}}}, t) \boldsymbol{B} \boldsymbol{A}(\boldsymbol{\eta}, \tilde{\boldsymbol{q}}, \dot{\tilde{\boldsymbol{q}}}, t)
$$

and

$$
\boldsymbol{C}_{\eta}(\boldsymbol{\eta}, \dot{\boldsymbol{\eta}}, \tilde{\boldsymbol{q}}, \dot{\tilde{\boldsymbol{q}}}, \ddot{\tilde{\boldsymbol{q}}}, t):=\boldsymbol{A}^{\mathrm{T}}(\boldsymbol{\eta}, \tilde{\boldsymbol{q}}, \dot{\tilde{\boldsymbol{q}}}, t) \boldsymbol{B} \dot{\boldsymbol{A}}(\boldsymbol{\eta}, \tilde{\boldsymbol{q}}, \dot{\tilde{\boldsymbol{q}}}, t) .
$$

We now choose the following control law

$$
\boldsymbol{u}=\check{\boldsymbol{u}}+\hat{\boldsymbol{u}}+\overline{\boldsymbol{u}} .
$$

\footnotetext{
${ }^{5}$ For the implementation in our robot system we use fixed point iteration. ${ }^{6}$ Higher derivatives of $\boldsymbol{q}$, such as $\ddot{\boldsymbol{q}}$ and $\boldsymbol{q}^{(3)}$, are calculated based on the model of the plant. In the end, the controller solely depends on $[\boldsymbol{\eta}, \tilde{\boldsymbol{q}}]$ and $[\dot{\boldsymbol{\eta}}, \dot{\tilde{\boldsymbol{q}}}]$. In order to better understand which terms depend in the first instance on higher derivatives, we do not make these substitutions.

${ }^{7}$ The singular values of $\kappa(\phi)$ are bounded and bounded away from zero. Thus, $\kappa^{-1}$ exists and is again bounded.
}

where

$$
\begin{aligned}
\check{\boldsymbol{u}} & :=\boldsymbol{B} \dot{\boldsymbol{a}}(\boldsymbol{\eta}, \tilde{\boldsymbol{q}}, \dot{\tilde{\boldsymbol{q}}}, \ddot{\tilde{\boldsymbol{q}}}, t) \\
\hat{\boldsymbol{u}} & :=\boldsymbol{\psi}(\boldsymbol{\theta}-\boldsymbol{q})-\boldsymbol{A}^{\mathrm{T}^{-1}} \boldsymbol{\psi}(\boldsymbol{\eta}-\tilde{\boldsymbol{q}}) \\
\overline{\boldsymbol{u}} & :=-\boldsymbol{A}^{\mathrm{T}^{-1}}\left(\boldsymbol{K}_{D} \dot{\boldsymbol{\eta}}+\boldsymbol{K}_{P} \boldsymbol{\eta}\right)
\end{aligned}
$$

which yields the following closed-loop motor dynamics

$$
M_{\eta}(\boldsymbol{\eta}, \tilde{\boldsymbol{q}}, \dot{\tilde{\boldsymbol{q}}}, t) \ddot{\boldsymbol{\eta}}+\boldsymbol{C}_{\eta}(\boldsymbol{\eta}, \dot{\boldsymbol{\eta}}, \tilde{\boldsymbol{q}}, \dot{\tilde{\boldsymbol{q}}}, \ddot{\tilde{\boldsymbol{q}}}, t) \dot{\boldsymbol{\eta}}+\boldsymbol{\psi}(\boldsymbol{\eta}-\tilde{\boldsymbol{q}})=\boldsymbol{A}^{\mathrm{T}} \overline{\boldsymbol{u}}
$$

Equation (21) resembles the one depicted in Fig. 2. This allows a physically intuitive interpretation of the resulting behavior.

As will be proven later, the PD controller $\overline{\boldsymbol{u}}$ in (20) globally, uniformly stabilizes the system. Thereby, the controller matrices have to meet the following conditions:

Assumption 5. The bounded controller gain matrices $\boldsymbol{K}_{P}, \boldsymbol{K}_{D} \in \mathbb{R}^{n \times n}$ are symmetric and positive definite. Note, $\boldsymbol{K}_{D}$ can also be a function of the states $\boldsymbol{\eta}, \tilde{\boldsymbol{q}}$.

By doing a full coordinate transformation, state-dependent virtual mass and Coriolis matrices naturally arise on the controlled motor side. The resulting closed-loop dynamics have some beneficial properties, such as the skew-symmetry of $\dot{M}_{\eta}-2 C_{\eta}$, which will be exploited later in the stability proof. Physically, this can be interpreted as energy conservation properties of the motor, i.e. the time derivative of the motors virtual kinetic energy $\dot{\boldsymbol{\eta}}^{\mathrm{T}} \boldsymbol{M}_{\eta} \dot{\boldsymbol{\eta}}$ is equal to the virtual power provided by the springs $\boldsymbol{\psi}(\boldsymbol{\eta}-\boldsymbol{q})$ and the PD control input $\bar{u}$

$$
\frac{1}{2} \frac{\mathrm{d}}{\mathrm{dt}}\left(\dot{\boldsymbol{\eta}}^{\mathrm{T}} \boldsymbol{M}_{\eta} \dot{\boldsymbol{\eta}}\right)=\dot{\boldsymbol{\eta}}^{\mathrm{T}}(-\boldsymbol{\psi}(\boldsymbol{\eta}-\tilde{\boldsymbol{q}})+\overline{\boldsymbol{u}}) .
$$

Skew symmetry of $\dot{M}_{\eta}-2 C_{\eta}$ can be shown easily. By observing

$$
\boldsymbol{B}=\boldsymbol{B}^{\mathrm{T}} \Longrightarrow \dot{\boldsymbol{M}}_{\eta}=\dot{\boldsymbol{A}}^{\mathrm{T}} \boldsymbol{B} \boldsymbol{A}+\boldsymbol{A}^{\mathrm{T}} \boldsymbol{B} \dot{\boldsymbol{A}}=\boldsymbol{C}_{\eta}^{\mathrm{T}}+\boldsymbol{C}_{\eta}
$$

one can easily show the skew symmetry

$$
\begin{aligned}
\boldsymbol{\eta}^{\mathrm{T}}\left(\dot{\boldsymbol{M}}_{\eta}-2 \boldsymbol{C}_{\eta}\right) \boldsymbol{\eta} & =\boldsymbol{\eta}^{\mathrm{T}}\left(\boldsymbol{C}_{\eta}^{\mathrm{T}}-\boldsymbol{C}_{\eta}\right) \boldsymbol{\eta}=\boldsymbol{\eta}^{\mathrm{T}}\left(-\boldsymbol{C}_{\eta}^{\mathrm{T}}+\boldsymbol{C}_{\eta}\right)^{\mathrm{T}} \boldsymbol{\eta} \\
& =-\boldsymbol{\eta}^{\mathrm{T}}\left(\boldsymbol{C}_{\eta}^{\mathrm{T}}-\boldsymbol{C}_{\eta}\right)^{\mathrm{T}} \boldsymbol{\eta} .
\end{aligned}
$$

Note, as we keep the structure and coupling of the original dynamics intact, we retain passivity properties which allows us to find a virtual energy-based Lyapunov function for the stability proof, see Sec. V.

\section{Quasi-rigid Manipulators}

The transition to the rigid robot case (i.e. $\sigma(\kappa) \rightarrow \infty$ ) yields the well-known PD+ controller [22].

\section{Stability \& Passivity Analysis}

In this section we analyze the passivity properties and derive a stability statement for the time-variant closed-loop 


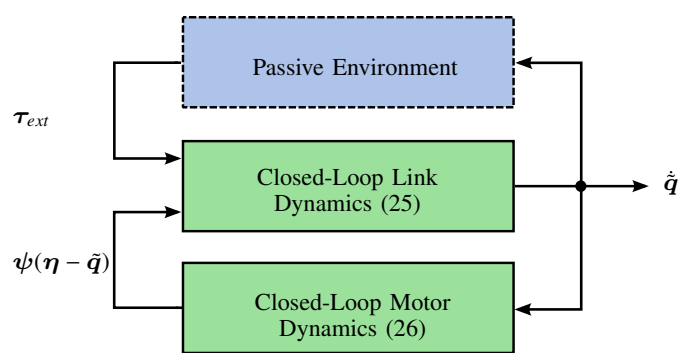

Fig. 3. The closed-loop dynamics (25)-(26) can be represented as an interconnection of passive subsystems. For the tracking case the link side interacts with the motor side via the power port $\dot{\tilde{\boldsymbol{q}}}^{\mathrm{T}} \boldsymbol{\psi}(\boldsymbol{\eta}-\tilde{\boldsymbol{q}})$ and with a passive environment via the power port $\dot{\tilde{\boldsymbol{q}}}^{\mathrm{T}} \boldsymbol{\tau}_{\text {ext }}$.

dynamics $^{8}$

$$
\begin{array}{r}
\boldsymbol{M}(\tilde{\boldsymbol{q}}, t) \ddot{\tilde{\boldsymbol{q}}}+C(\tilde{\boldsymbol{q}}, \dot{\tilde{\boldsymbol{q}}}, t) \dot{\tilde{\boldsymbol{q}}}=-D \dot{\boldsymbol{q}}+\psi(\boldsymbol{\eta}-\tilde{\boldsymbol{q}})+\tau_{e x t}, \\
M_{\eta}(\boldsymbol{\eta}, \tilde{\boldsymbol{q}}, \dot{\tilde{\boldsymbol{q}}}, t) \ddot{\boldsymbol{\eta}}+C_{\eta}(\boldsymbol{\eta}, \dot{\boldsymbol{\eta}}, \tilde{\boldsymbol{q}}, \dot{\tilde{\boldsymbol{q}}}, \ddot{\boldsymbol{q}}, t) \dot{\boldsymbol{\eta}}+\psi(\boldsymbol{\eta}-\tilde{\boldsymbol{q}})=\overline{\boldsymbol{u}}
\end{array}
$$

\section{A. Passivity}

We proove that the link-side dynamics (25) and motor dynamics (26) of the closed-loop system can be interpreted as an interconnection of passive subsystems, see Fig. 3. A according to the definition in [26], we have to show that for any period of time, the system cannot output more virtual energy at its ports of interaction than has in total been put into for that period of time. With Fig. 2.c in mind it is natural to choose

$$
S_{\tilde{q}}:=\frac{1}{2} \dot{\tilde{\boldsymbol{q}}}^{\mathrm{T}} \boldsymbol{M}(\tilde{\boldsymbol{q}}, t) \dot{\tilde{\boldsymbol{q}}}
$$

as storage function for the closed-loop link-side dynamics (25) and

$$
S_{\eta}:=\frac{1}{2} \dot{\boldsymbol{\eta}}^{\mathrm{T}} \boldsymbol{M}_{\eta}(\boldsymbol{\eta}, \tilde{\boldsymbol{q}}, \dot{\tilde{\boldsymbol{q}}}, t) \dot{\boldsymbol{\eta}}+U_{s}(\boldsymbol{\eta}-\tilde{\boldsymbol{q}})+\frac{1}{2} \boldsymbol{\eta}^{\mathrm{T}} \boldsymbol{K}_{P} \boldsymbol{\eta},
$$

as storage function for the closed-loop motor dynamics (26). Eq. (27) represents the virtual kinetic energy of the link. Accordingly, the first term on the RHS in (28) represents the virtual kinetic energy of the controlled motor. The other two terms can be interpreted as the potential energy of the spring and a virtual potential energy of the control, respectively.

For the analysis of the passivity properties, we express the time derivative of (27) along the solutions of (25). By exploiting Prop. 3 we can write

$$
\dot{S}_{\tilde{q}}=-\dot{\tilde{\boldsymbol{q}}}^{\mathrm{T}} \boldsymbol{D} \dot{\tilde{\boldsymbol{q}}}+\dot{\tilde{\boldsymbol{q}}}^{\mathrm{T}} \boldsymbol{\psi}(\boldsymbol{\eta}-\tilde{\boldsymbol{q}})+\dot{\tilde{\boldsymbol{q}}}^{\mathrm{T}} \boldsymbol{\tau}_{\text {ext }} .
$$

We can identify three terms in (29). The first one represents the dissipation of energy due to the damping assignment on the link side. The latter two are each corresponding to an interconnection port with the link dynamics. As visualized in Fig. $3 \dot{\tilde{\boldsymbol{q}}}^{\mathrm{T}} \boldsymbol{\psi}(\boldsymbol{\eta}-\tilde{\boldsymbol{q}})$ represents the interconnection with the motor side and $\dot{\tilde{\boldsymbol{q}}}^{\mathrm{T}} \boldsymbol{\tau}_{\text {ext }}$ represents the interconnection with the environment. Correspondingly, by exploiting the skew symmetry of $\dot{M}_{\eta}-2 C_{\eta}$, we can write the time derivative of (27) as follows

$$
\dot{S}_{\eta}=-\dot{\boldsymbol{\eta}}^{\mathrm{T}} \boldsymbol{K}_{D} \dot{\boldsymbol{\eta}}-\dot{\tilde{\boldsymbol{q}}}^{\mathrm{T}} \boldsymbol{\psi}(\boldsymbol{\eta}-\tilde{\boldsymbol{q}}) .
$$

\footnotetext{
${ }^{8}$ Recall, the coordinate transformation (7) imposes equality of (1) and (25).
}

Again, we can identify a dissipation term. This term is related to the introduced damping on the motor side. The interconnection term also appears again (cf. (29)). The analysis so far motivates the following proposition:

Proposition 1. The closed-loop system (25)-(26) is a passive map from the generalized external forces $\tau_{\text {ext }}$ to the generalized velocity of the link-side tracking error $\dot{\tilde{\boldsymbol{q}}}$.

Proof. Consider the storage function $S=S_{\tilde{q}}+S_{\eta}$ comprising (27) and (28). Its time derivative is given by the sum of (29) and (30)

$$
\dot{S}=-\dot{\tilde{\boldsymbol{q}}}^{\mathrm{T}} \boldsymbol{D} \dot{\tilde{\boldsymbol{q}}}+\dot{\tilde{\boldsymbol{q}}} \tau_{e x t}-\dot{\boldsymbol{\eta}}^{\mathrm{T}} \boldsymbol{K}_{D} \dot{\boldsymbol{\eta}} \leq \dot{\tilde{\boldsymbol{q}}}^{\mathrm{T}} \boldsymbol{\tau}_{\text {ext }},
$$

which completes the proof.

Note, for the regulation case passivity with respect to the physically more intuitive power port $\dot{\boldsymbol{q}}, \boldsymbol{\tau}_{\text {ext }}$ is given. Nevertheless, situations may arise in practice where passivity with respect to the power port $\dot{\tilde{\boldsymbol{q}}}, \tau_{\text {ext }}$ will be of importance. More specifically, there exist situations where the environment or the interacting object move synchronous to the link reference trajectory. One such scenario would be object manipulation on a conveyor belt.

\section{B. Stability}

The physically motivated storage function from the passivity analysis, which is based on the intuitive representation of the closed-loop dynamics in Fig. 2, motivates the following:

Proposition 2. For $\tau_{\text {ext }}=\mathbf{0}$, the closed-loop dynamics (25)(26) is globally, uniformly stable under the assumptions made in Sec. III.

Proof. First, we will show stability by invoking Lyapunov's direct method for non-autonomous systems [27]. For that we have to show the existence of a positive definite, scalar function $V$ with continuous partial derivatives such that $\dot{V}$ is negative semi-definite. By showing the existence of a timeinvariant upper bound $V^{*}$ such that $V^{*}(\boldsymbol{x}) \geq V(\boldsymbol{x}, t) \geq 0, \forall t$, we can extend that statement to uniform stability. Consider

$$
V(\boldsymbol{\eta}, \dot{\boldsymbol{\eta}}, \tilde{\boldsymbol{q}}, \dot{\tilde{\boldsymbol{q}}}, t)=S_{\eta}(\boldsymbol{\eta}, \dot{\boldsymbol{\eta}}, \tilde{\boldsymbol{q}}, \dot{\tilde{\boldsymbol{q}}}, t)+S_{\tilde{q}}(\boldsymbol{\eta}, \dot{\boldsymbol{\eta}}, \tilde{\boldsymbol{q}}, \dot{\tilde{\boldsymbol{q}}}, t)
$$

as an virtual-energy-based Lyapunov function candidate. The positive definiteness of $V$ follows directly from the assumption made in Sec. III.

The time derivative of $V$ along the solutions of (25)-(26)

$$
\dot{V}=-\dot{\tilde{\boldsymbol{q}}}^{\mathrm{T}} \boldsymbol{D} \dot{\tilde{\boldsymbol{q}}}-\dot{\boldsymbol{\eta}}^{\mathrm{T}} \boldsymbol{K}_{D} \dot{\boldsymbol{\eta}}
$$

is non-positive, recall $\boldsymbol{K}_{D}, \boldsymbol{D}>0$ according to the Ass. 4 and 5. Thus, (32) is a Lyapunov function. A time-invariant upper bound for $V$ is given by

$$
V^{*}=\frac{1}{2} \dot{\tilde{\boldsymbol{q}}}^{\mathrm{T}} \overline{\boldsymbol{M}} \dot{\tilde{\boldsymbol{q}}}+\frac{1}{2} \dot{\boldsymbol{\eta}}^{\mathrm{T}} \overline{\boldsymbol{M}}_{\eta} \dot{\boldsymbol{\eta}}+U_{s}(\boldsymbol{\eta}-\tilde{\boldsymbol{q}})+\boldsymbol{\eta}^{\mathrm{T}} \boldsymbol{K}_{P} \boldsymbol{\eta}
$$

where we exploit the fact that $\boldsymbol{M}$ is uniformly bounded according to [25] and that $\boldsymbol{M}_{\eta}$ is uniformly bounded as well. The latter can be proven by applying the following lemma:

Lemma 1 (J. K. Merikoski and R. Kumar, [28]). If $\boldsymbol{A}$ and $\boldsymbol{B}$ are Hermitian and non-negative definite, then we can both 
underestimate and overestimate eigenvalues of $\boldsymbol{A} \boldsymbol{B}$ by using eigenvalues of $\boldsymbol{A}$ and $\boldsymbol{B}$.

$$
\lambda_{i}(\boldsymbol{A}) \lambda_{\min }(\boldsymbol{B}) \leq \lambda_{i}(\boldsymbol{A} \boldsymbol{B}) \leq \lambda_{i}(\boldsymbol{A}) \lambda_{\max }(\boldsymbol{B}) .
$$

By writing $M_{\eta}$ as a product of two positive definite Hermitian matrices and applying the lemma above, we can derive the following inequalities 9

$$
\begin{aligned}
\lambda_{\max }\left(\boldsymbol{M}_{\eta}\right) & =\lambda_{\max }\left(\boldsymbol{A}^{\mathrm{T}} \boldsymbol{B} \boldsymbol{A}\right)=\lambda_{\max }\left(\left(\boldsymbol{A}^{\mathrm{T}} \boldsymbol{B}^{1 / 2}\right)\left(\boldsymbol{A}^{\mathrm{T}} \boldsymbol{B}^{1 / 2}\right)^{\mathrm{T}}\right) \\
& \leq \lambda_{\max }^{2}\left(\boldsymbol{A}^{\mathrm{T}} \boldsymbol{B}^{1 / 2}\right) \leq \lambda_{\max }^{2}(\boldsymbol{A}) \lambda_{\max }(\boldsymbol{B}) .
\end{aligned}
$$

The second inequality requires applying the lemma a second time. For that $\boldsymbol{A}^{\mathrm{T}}$ and $\boldsymbol{B}^{1 / 2}$ both have to be Hermitian and non-negative definite. Since $\kappa(\phi)$ is derived via a positive definite potential function, see Ass. $1, \kappa$ is symmetric and positive definite. Therefore, the same is true for $\boldsymbol{A}$, (cf. (12)). For $\boldsymbol{B}$ the conditions are obviously true, recall Prop. 2. Under Ass. $1, \boldsymbol{\kappa}(\boldsymbol{\eta}-\tilde{\boldsymbol{q}})$ is bounded for bounded $\tilde{\boldsymbol{q}}$ and $\boldsymbol{\eta}$, which has been shown above, thus $\boldsymbol{A}$ is bounded. This, together with the fact that $\boldsymbol{B}$ is constant leads to the boundedness of $\bar{M}_{\eta}$.

\section{Convergence of velocities}

To show convergence of the velocities for the closed-loop system (25)-(26) in case $\boldsymbol{\tau}_{\text {ext }}=\mathbf{0}$, i.e., $\dot{\boldsymbol{q}}-\dot{\boldsymbol{q}}_{d}(t) \rightarrow \mathbf{0}$ and $\dot{\boldsymbol{\eta}} \rightarrow \mathbf{0}$ as $t \rightarrow \infty$, in case $\boldsymbol{\tau}_{\text {ext }}=0$, we apply Barbalat's lemma [27].

Proof. We choose the same Lyapunov function $V$ as for the stability proof. As we have already shown that $V(\boldsymbol{\eta}, \dot{\boldsymbol{\eta}}, \tilde{\boldsymbol{q}}, \dot{\tilde{\boldsymbol{q}}}, t)$ is lower bounded and that $\dot{V}(\boldsymbol{\eta}, \dot{\boldsymbol{\eta}}, \tilde{\boldsymbol{q}}, \dot{\tilde{\boldsymbol{q}}}, t)$ is negative semidefinite, what is left to show is that $\dot{V}(\boldsymbol{\eta}, \dot{\boldsymbol{\eta}}, \tilde{\boldsymbol{q}}, \dot{\tilde{\boldsymbol{q}}}, t)$ is uniformly continuous in time. A sufficient condition for uniform continuity is that $\ddot{V}(\boldsymbol{\eta}, \dot{\boldsymbol{\eta}}, \tilde{\boldsymbol{q}}, \dot{\tilde{\boldsymbol{q}}}, t)$ is bounded. To this end, we derive $\dot{V}$ along the solutions of (25)-(26)

$$
\ddot{V}(\boldsymbol{\eta}, \dot{\boldsymbol{\eta}}, \tilde{\boldsymbol{q}}, \dot{\tilde{\boldsymbol{q}}}, t)=-2 \dot{\tilde{\boldsymbol{q}}}^{\mathrm{T}} \boldsymbol{D} \ddot{\tilde{\boldsymbol{q}}}-\dot{\tilde{\boldsymbol{q}}}^{\mathrm{T}} \dot{\boldsymbol{D}} \dot{\tilde{\boldsymbol{q}}}-2 \dot{\boldsymbol{\eta}}^{\mathrm{T}} \boldsymbol{K}_{D} \ddot{\boldsymbol{\eta}}-\dot{\boldsymbol{\eta}}^{\mathrm{T}} \dot{\boldsymbol{K}}_{D} \dot{\boldsymbol{\eta}}
$$

With the bounds we have already established above, it is easy to verify the boundedness of $\ddot{V}$. Recall, the singular values of $M$ are bounded and bounded away from zero, thus $\ddot{\tilde{q}}$ is bounded. From that, Ass. 1 and (26) it follows directly the boundedness of $\ddot{\eta}$. Thus, all terms in (37) are bounded and all conditions of Barbalat's Lemma are satisfied. This implies $\dot{V}(\boldsymbol{\eta}, \dot{\boldsymbol{\eta}}, \tilde{\boldsymbol{q}}, \dot{\tilde{\boldsymbol{q}}}, t) \rightarrow 0$ for $t \rightarrow \infty$, which, together with (33), completes the proof.

\section{Experimental Validation and Performance Analysis}

The proposed control law has been experimentally evaluated on the first four joints of the DLR variable stiffness robot arm called the Hand Arm System. These four joints, namely the elbow and the three shoulder joints, are implemented by Floating Spring Joints (FSJ) [30] which belong to the class of Variable Stiffness Actuators (VSA). All four joints feature highly nonlinear elasticities, see Fig. 1.

\footnotetext{
${ }^{9}$ Note, a positive semi-definite matrix has precisely one positive semidefinite square root, see [29]. From the spectral theorem follows that $\lambda_{i}\left(B^{1 / 2}\right)=\lambda^{1 / 2}(B)$.
}

\section{A. Design of Link- and Motor-Side Damping}

As the inertia of the robot system is configuration dependent, it varies significantly throughout its workspace. In addition the stiffness of each joint strongly depends on the load. As the robot moves through its workspace, the gravitational and dynamical load change constantly and so do the joints stiffness's. External forces may additionally affect the stiffness's of the joints. As we aim for similar performance throughout the entire workspace of the robot, we apply a damping design that takes these effects into account. The design of the link-side damping matrix $D$ as well as the controller gain $\boldsymbol{K}_{D}$ are based on modal decomposition. Consider the linearized system

$$
\boldsymbol{M}(\boldsymbol{q}) \ddot{\tilde{\boldsymbol{q}}}+\boldsymbol{D}(\boldsymbol{\eta}, \tilde{\boldsymbol{q}}) \dot{\tilde{\boldsymbol{q}}}-\boldsymbol{\kappa}(\boldsymbol{\eta}-\tilde{\boldsymbol{q}})(\boldsymbol{\eta}-\tilde{\boldsymbol{q}})=0
$$

of the closed-loop link dynamics. We derive $D$ from the corresponding generalized eigenvalue problem such that

$$
\boldsymbol{D}(\boldsymbol{\eta}, \tilde{\boldsymbol{q}})=2 \boldsymbol{Q}(\boldsymbol{\eta}, \tilde{\boldsymbol{q}})^{-\mathrm{T}} \operatorname{diag}\left(\xi_{D, i} \sqrt{\lambda_{i}}\right) \boldsymbol{Q}(\boldsymbol{\eta}, \tilde{\boldsymbol{q}})^{-1},
$$

where $\xi_{i}$ are modal damping factors. $Q \in \mathbb{R}^{n \times n}$ is a nonsingular matrix satisfying $\boldsymbol{Q}^{\mathrm{T}} \boldsymbol{Q}=\boldsymbol{M}$ and $\kappa=Q^{\mathrm{T}} \Lambda \boldsymbol{Q}$, where $\Lambda$ is a diagonal matrix of the real, positive eigenvalues $\lambda_{i}$. This method relies on the fact that $M$ is positive definite and that $\kappa$ is symmetric. See [21] for more information. For the design of $\boldsymbol{K}_{D}$ we consider the linearized system

$$
\boldsymbol{M}_{\boldsymbol{\eta}}(\boldsymbol{\eta}, \tilde{\boldsymbol{q}}, \dot{\tilde{\boldsymbol{q}}}, t) \ddot{\boldsymbol{\eta}}+\boldsymbol{K}_{D} \dot{\boldsymbol{\eta}}+\boldsymbol{\kappa}(\boldsymbol{\eta}-\tilde{\boldsymbol{q}})(\boldsymbol{\eta}-\tilde{\boldsymbol{q}})+\boldsymbol{K}_{P} \boldsymbol{\eta}
$$

and proceed anaglogously to above, resulting in a statedependent $\boldsymbol{K}_{D}$.

Remark 1. Stability for a state-dependent damping matrix $\boldsymbol{D}(\boldsymbol{\eta}, \tilde{\boldsymbol{q}})$ has been only experimentally verified so far. From a theoretical point of view one would have to show that (7) represents a diffeomorphism between $\boldsymbol{\theta}$ and $\boldsymbol{\eta}$ even for a damping matrix that is a function of $\boldsymbol{\eta}$ and $\tilde{\boldsymbol{q}}$.

\section{B. Experimental results}

Throughout all experiments the controller parameters were set as follows: $\boldsymbol{K}_{P}=\operatorname{diag}(1000,900,750,750), \xi_{D, i}=0.5$, $\xi_{K_{D}, i}=0.7, i=1, \ldots, 4 . \boldsymbol{K}_{P}$ was manually set such that none of the control inputs $(\boldsymbol{u})$ overshoots the maximal motor torques of $65 \mathrm{Nm} .{ }^{10}$ For all joints the stiffness setting was set to the medium value of $\sigma=5 \mathrm{deg}$, which corresponds to the central curve of Fig. 1c. Two kind of experiments were performed.

The first experiment shows the tracking performance, see Fig. VI-B. During the trajectory, the joints reach a maximum speed of $\dot{\boldsymbol{q}}^{\mathrm{T}}=[190,73,103,114] \mathrm{deg} / \mathrm{s}$. Due to the swift motion, the transient tracking performance is limited by unmodeled velocity-dependent friction. We use no friction compensation here. Obviously, increasing $\boldsymbol{K}_{P}$ would lower the tracking error at the cost of increased control inputs $\boldsymbol{u}$.

The second experiment was set up such that a $3 \mathrm{~kg}$ mass attached to a rope hits the robot in a swinging motion in a

\footnotetext{
${ }^{10}$ In standard operation mode saturation of the control input hasn't caused any issues so far, nevertheless to allow a fair performance analysis the gains were such that saturation effects are avoided.
} 
reproducible manner, see Fig. VI-B. The robot is commanded to keep its pre impact position. Within approx. $0.2 \mathrm{~s}$ post impact the system converges into an $\pm 1 \mathrm{deg}$ error band. To show the elasticity and oscillatory behavior of the joints the performance of a simple motor PD controller is shown as reference.

Compared to our previous approach [24], our new approach presented here requires less control action (lower amplitudes) and has a smoother transient behavior.

A video of the corresponding experiments can be found under: http://www.dlr.de/rmc/rm/en/desktopdefault.aspx/tabid-5486/.
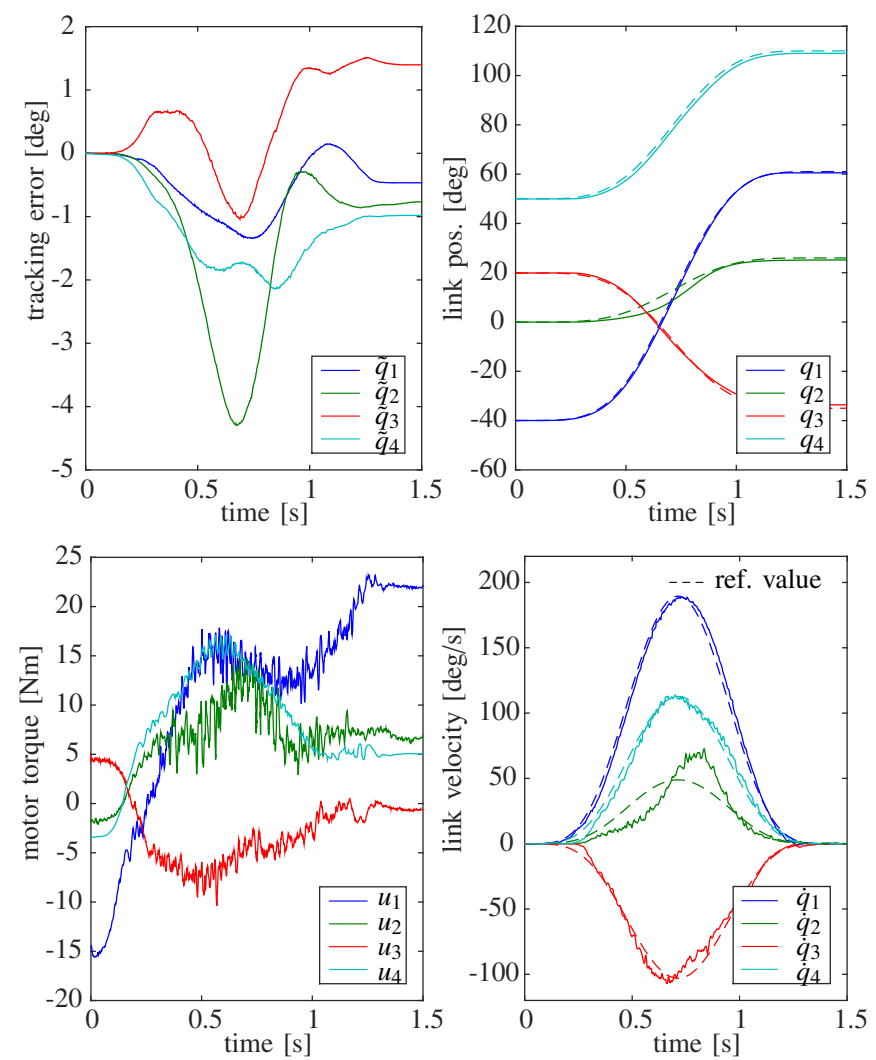

Fig. 4. The tracking performance is shown on the DLR Hand Arm System. The trajectory was chosen such that strong coupling effects between the joints are present. The time constant of the trajectory is $0.6 \mathrm{~s}$.

\section{Conclusion}

The controller developed in this paper is a globally stable link-side tracking controller that allows simultaneous linkside damping assignment for compliantly actuated robots with nonlinear elastic transmissions. The approach follows the idea of changing the original link as well as the motor dynamics only to a minimal extent.

A comprehensive passivity and stability analysis is given based on a physically motivated Lyapunov function. The controller concept imposes neither lower nor upper bounds on the controller gains. The transition to the rigid robot case, i.e., when the stiffness of each joint approaches infinity, yields the well-known PD+ controller [22] and thus no highgain design. We evaluated the approach experimentally on a multi-dof VSA robot with highly nonlinear elasticities. As any link-side tracking controller, the approach requires the second and third derivatives of the measured link coordinates. However, in practice this did not turn out to be a problem since the time derivatives could be calculated based on a dynamics model of the robot. The high compliance of the joint allows an accurate and high resolution measurement of the joint deflection, thus model based estimations of higher derivations cause no significant problems.

While stability was shown in this paper, the extension of the analysis to asymptotic stability is topic of our current research. Another issue we consider worth investigating is to check whether the theory presented here can be extended to allow for state-dependent link side damping in the controller.

\section{APPENDIX}

Using (7) we can write

$$
\begin{aligned}
\boldsymbol{\kappa}(\boldsymbol{\theta}-\boldsymbol{q})= & \boldsymbol{\kappa}\left(\boldsymbol{\psi}^{-1}(\boldsymbol{\psi}(\boldsymbol{\eta}-\tilde{\boldsymbol{q}}))+\boldsymbol{g}\left(\tilde{\boldsymbol{q}}+\boldsymbol{q}_{d}(t)\right)\right. \\
& \left.-\boldsymbol{D} \dot{\tilde{\boldsymbol{q}}}+\boldsymbol{n}_{0}(\tilde{\boldsymbol{q}}, \dot{\tilde{\boldsymbol{q}}}, t)\right)=: \chi(\boldsymbol{\eta}, \tilde{\boldsymbol{q}}, \dot{\tilde{\boldsymbol{q}}}, t) .
\end{aligned}
$$

This eq. together with (10) allows us to define $\boldsymbol{A}:=\chi^{-1}(\boldsymbol{\eta}, \tilde{\boldsymbol{q}}, \dot{\tilde{\boldsymbol{q}}}, t) \boldsymbol{\kappa}(\boldsymbol{\eta}-\tilde{\boldsymbol{q}})$ and $\boldsymbol{a}:=\tilde{\boldsymbol{q}}+\boldsymbol{q}_{d}(t)+$ $\chi^{-1}(\boldsymbol{\eta}, \tilde{\boldsymbol{q}}, \dot{\tilde{\boldsymbol{q}}}, t) \gamma(\boldsymbol{\eta}, \tilde{\boldsymbol{q}}, \dot{\tilde{\boldsymbol{q}}}, \ddot{\tilde{\boldsymbol{q}}}, t)$.

\section{REFERENCES}

[1] G. A. Pratt and M. M. Williamson, "Series elastic actuators," in Intelligent Robots and Systems 95.'Human Robot Interaction and Cooperative Robots', Proceedings. 1995 IEEE/RSJ International Conference on, vol. 1. IEEE, 1995, pp. 399-406.

[2] P. Tomei, "A simple pd controller for robots with elastic joints," Automatic Control, IEEE Transactions on, vol. 36, no. 10, pp. 12081213, Oct 1991.

[3] R. Ortega, R. Kelly, and A. Loria, "A class of output feedback globally stabilizing controllers for flexible joints robots," Robotics and Automation, IEEE Transactions on, vol. 11, no. 5, pp. 766-770, Oct 1995.

[4] L. Zollo, A. De Luca, and B. Siciliano, "Regulation with on-line gravity compensation for robots with elastic joints," in Robotics and Automation, 2004. Proceedings. ICRA '04. 2004 IEEE International Conference on, vol. 3, April 2004, pp. 2687-2692.

[5] C. Ott, A. Albu-Schäffer, A. Kugi, S. Stamigioli, and G. Hirzinger, "A passivity based cartesian impedance controller for flexible joint robots-part i: Torque feedback and gravity compensation," in Proc. IEEE Int. Conf. on Robotics and Automation, 2004.

[6] B. Vanderborght, A. Albu-Schäffer, A. Bicchi, E. Burdet, D. G. Caldwell, R. Carloni, M. Catalano, O. Eiberger, W. Friedl, G. Ganesh et al., "Variable impedance actuators: A review," Robotics and Autonomous Systems, vol. 61, no. 12, pp. 1601-1614, 2013.

[7] A. Albu-Schäffer, C. Ott, and F. Petit, "Constructive energy shaping control for a class of euler-lagrange systems," in 10th Int. IFAC Symposium on Robot Control, 2012.

[8] A. Albu-Schäffer and G. Hirzinger, "A globally stable state feedback controller for flexible joint robots," Advanced Robotics, vol. 15, no. 8, pp. 799-814, 2001.

[9] F. Petit and A. Albu-Schäffer, "State feedback damping control for a multi dof variable stiffness robot arm," in Proc. IEEE Int. Conf. on Robotic and Automation, 2011.

[10] I. Sardellitti, G. Medrano-Cerda, N. Tsagarakis, A. Jafari, and D. Caldwell, "Gain scheduling control for a class of variable stiffness actuators based on lever mechanisms," Robotics, IEEE Transactions on, vol. 29, no. 3, pp. 791-798, June 2013.

[11] M. W. Spong, "Modeling and control of elastic joint robots," Transactions of the ASME: Journal of Dynamic Systems, Measurement, and Control, vol. 109, pp. 310-319, 1987.

[12] S. Nicosia and P. Tomei, "Design of global tracking controllers for flexible-joint robots," Journal of robotic systems, vol. 10, no. 6, pp. 835-846, 1993.

[13] A. Loria and R. Ortega, "On tracking control of rigid and flexible joints robots," Appl. Math. Comput. Sci, vol. 5, no. 2, pp. 101-113, 1995.

[14] C. Ott, A. Albu-Schäffer, A. Kugi, and G. Hirzinger, "Decoupling based cartesian impedance control of flexible joint robots," in Proc. IEEE Int. Conf. on Robotics and Automation, 2003. 

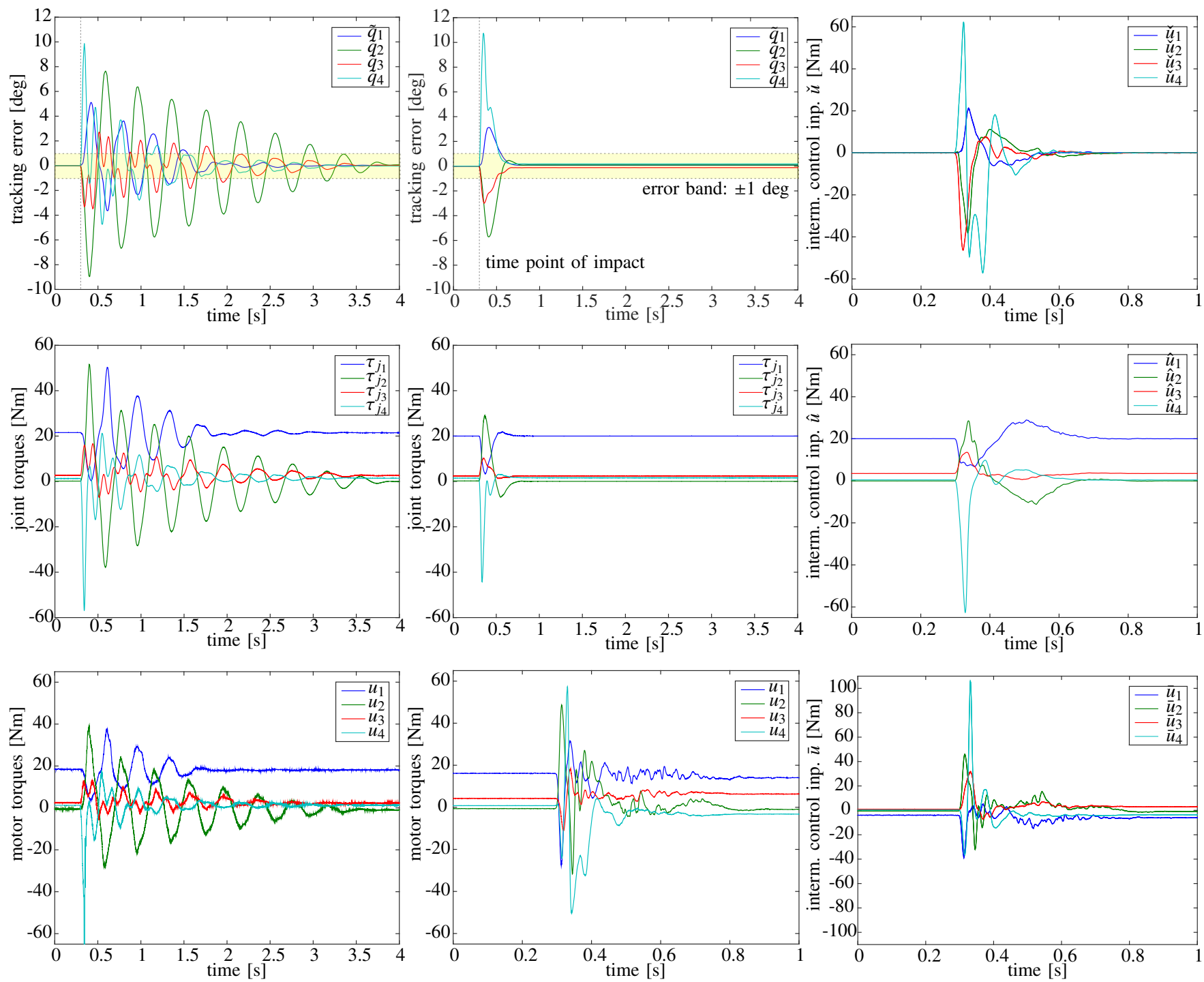

Fig. 5. This experiment shows the damping performance of the proposed controller (center and right column) on the DLR Hand Arm System. As reference, to show the oscillatory nature of the system, the performance of a standard motor PD controller is shown in comparison (left column). During the experiment the robot is commanded to hold its link positions while it is hit, in reproducible manner, with a $3 \mathrm{~kg}$ mass that swings on a rope. For the proposed controller, the systems link-side errors $\tilde{q}_{i}$ converge within approx. $0.2 \mathrm{~s}$ post impact into an \pm 1 deg error band.

[15] J. H. Oh and J. S. Lee, "Control of flexible joint robot system by backstepping design approach," Intelligent Automation $\mathcal{E}$ Soft Computing, vol. 5, no. 4, pp. 267-278, 1999.

[16] C. Ott, Cartesian Impedance Control of Redundant and Flexible-Joint Robots, B. Siciliano and O. Khatib, Eds. Springer, 2008.

[17] J.-J. Slotine and L. Weiping, "Adaptive manipulator control: A case study," Automatic Control, IEEE Transactions on, vol. 33, no. 11, pp. 995-1003, 1988

[18] M. W. Spong, "Adaptive control of flexible joint manipulators," Systems $\mathcal{E}$ Control Letters, vol. 13, no. 1, pp. 15-21, 1989.

[19] A. De Luca and P. Lucibello, "A general algorithm for dynamic feedback linearization of robots with elastic joints," in IEEE Int. Conf. on Robotics and Automation, vol. 1, 1998, pp. 504-510.

[20] G. Palli, C. Melchiorri, and A. De Luca, "On the feedback linearization of robots with variable joint stiffness," in IEEE Int. Conf. on Robotics and Automation, 2008, pp. 1753-1759.

[21] C. Ott, A. Kugi, and Y. Nakamura, "Resolving the problem of non-integrability of nullspace velocities for compliance control of redundant manipulators by using semi-definite Lyapunov functions," in Proceedings IEEE International Conference on Robotics and Automation, Pasadena, USA, May 2008, pp. 1456-1463.

[22] B. Paden and R. Panja, "Globally asymptotically stable pd+controller for robot manipulators," International Journal of Control, vol. 47, no. 6, pp. $1697-1712,1988$.

[23] B. Brogliato, R. Ortega, and R. Lozano, "Global tracking controllers for flexible-joint manipulators: A comparative study," Automatica, vol. 31, no. 7, pp. 941-956, Jul. 1995.

[24] M. Keppler, D. Lakatos, C. Ott, and A. Albu-Schäffer, "A passivitybased approach for trajectory tracking and link-side damping of compliantly actuated robots," in 2016 IEEE International Conference on Robotics and Automation (ICRA). IEEE, 2016, pp. 1079-1086.

[25] F. Ghorbel, B. Srinivasan, and M. W. Spong, "On the uniform boundedness of the inertia matrix of serial robot manipulators," Journal of Robotic Systems, vol. 15, no. 1, 1998.

[26] A. van der Schaft, L2-gain and passivity techniques in nonlinear control. London New York: Springer, 2000.

[27] H. K. Khalil, Nonlinear Systems (3rd Edition). Prentice Hall, 2001

[28] J. K. Merikoski and R. Kumar, "Inequalities for spreads of matrix sums and products." Applied Mathematics E-Notes, vol. 4, pp. 150159, 2004.

[29] R. A. Horn and C. R. Johnson, Matrix Analysis. Cambridge University Press, 2012

[30] S. Wolf, O. Eiberger, and G. Hirzinger, "The dlr fsj: Energy based design of a variable stiffness joint," in Robotics and Automation (ICRA), 2011 IEEE International Conference on, May 2011, pp. 50825089. 